\title{
Chemically active substitutional nitrogen impurity in carbon nanotubes
}

\author{
Andriy H. Nevidomskyy, Gábor Csányi, and Michael C. Payne \\ Theory of Condensed Matter group, Cavendish Laboratory, \\ University of Cambridge, Cambridge CB3 OHE, UK
}

(Dated: October 20, 2018)

\begin{abstract}
We investigate the nitrogen substitutional impurity in semiconducting zigzag and metallic armchair single-wall carbon nanotubes using ab initio density functional theory. At low concentrations (less than 1 at. \%), the defect state in a semiconducting tube becomes spatially localized and develops a flat energy level in the band gap. Such a localized state makes the impurity site chemically and electronically active. We find that if two neighbouring tubes have their impurities facing one another, an inter-tube covalent bond forms. This finding opens an intriguing possibility for tunnel junctions, as well as the functionalization of suitably doped carbon nanotubes by selectively forming chemical bonds with ligands at the impurity site. If the intertube bond density is high enough, highly packed bundle of interlinked single-wall nanotubes can form.
\end{abstract}

PACS numbers: 61.46.+w, 81.07.De, 73.22.-f

Ever since their discovery 1, 2], carbon nanotubes (CNT) have been heralded as the new wonder-material of the future. Their remarkable mechanical and electronic properties destine them to play a major role in all kinds of nanotechnologies and molecular electronics. At least two major hurdles have to be overcome in order to fulfill this potential. First, manipulation of individual tubes is at best difficult today, which prevents mass production of devices. Second, the ability to fine-tune the various properties of the material to suit particular applications has to be achieved.

Carbon nanotubes exhibit a variety of electronic properties, for example, depending on their diameter and chirality, they vary from being metallic to semiconducting [3]. The presence of defects and impurities that are electronically or chemically active can change these properties and thus have a significant bearing on a broad range of applications. In this paper we investigate the nitrogen substitutional impurity in singlewall carbon nanotubes (SWNT). In the case of semiconducting tubes, doping with electrons or holes is the principle route to making electronic devices. Alternatively, introducing new levels in the band gap with associated electronic states that are spatially localized, can create chemically active impurity sites.

Several groups have produced nanotubes containing nitrogen 4, 5, 6, 7, 8, 9, 10, 11, 12, 13, 14] or nitrogen and boron [5, 15, 16]. All of these were multi wall tubes, except for [16], where nitrogen was always accompanied by boron. The measured atomic concentrations of the impurities were relatively high in all cases, above $1 \%$, and all the $\mathrm{CN}_{x}$ tubes were found to be metallic, as predicted by theoretical models 10, 11, 17. Indeed, one other reason for the particular interest in $\mathrm{CN}_{x}$ tubes was that they might make even initially semiconducting tubes metallic.

Various structural models have been proposed for the incorporation of nitrogen into the carbon network. Direct substitution was first studied by Yi and Bernholc 18 using density functional theory at an atomic impurity concentration of about 1\%, where they found an impurity state lying $0.27 \mathrm{eV}$ below the bottom of the conduction band at the $\Gamma$ point and a significant overlap of the states associated with the adjacent nitrogens $(8.4 \AA$ apart). Lammert et al. 19] considered disordered substitution of boron and nitrogen at a concentration of $3.5 \%$ and $1.4 \%$, and observed both donor and acceptor levels in the gap. For an isolated nitrogen or boron impurity, they found the impurity state to be localized within about $10 \AA$ for an $(8,0)$ tube.

Another atomic arrangement for the experimentally observed nitrogen impurity was proposed by Terrones et al. [6], where they suggest that at high concentrations (above about 10\%) nitrogens are divalent, pyridine-like. Later publications from the same group refine this model and correlate the calculated density of states of metallic tubes with experimental observations [10, 11].

To study the isolated substitutional nitrogen defect, the simulation cell has to be as long as possible. The existing constraints of computational power mean that the diameter of the tube should therefore be as small as possible. In all calculations we used the achiral zigzag $(8,0)$ and armchair $(5,5)$ nanotubes. Our simulation cell has periodic boundary conditions, the tubes are arranged parallel to the $\mathrm{z}$ axis and form a triangular lattice in the perpendicular $\mathrm{x}-\mathrm{y}$ plane. This arrangement is experimentally seen in carbon nanotube ropes 3$]$. We studied a range of nitrogen concentrations from $0.26 \%$ to $1 \%$ by introducting one impurity atom and changing the size of the simulation cell along the axis of the tube. The largest simulation cell contained 12 unit cells along the $\mathrm{z}$ axis for the zigzag tube and 14 unit cells for the armchair tube, respectively, with one impurity atom in it.

Throughout, we used ab-initio plane-wave pseudopotential density functional theory 20 as implemented in the CASTEP code 21]. The generalized gradient approximation was used to account for exchange and correlation 
in the Perdew-Burke-Ernzerhof form 22]. All calculations were spin-polarized and ultrasoft pseudopotentials were used for carbon and nitrogen with a cutoff energy of $300 \mathrm{eV}$. We assessed the adequacy of this cutoff by performing calculations at $350 \mathrm{eV}$ for the smallest unit cells and verified that none of properties reported in this work changed perceptibly. For each concentration, the ionic positions were optimized by using one k-point at the $\Gamma$ point for the largest simulation cells, and correspondingly more $\mathrm{k}-$ points for the smaller cells. After the geometry optimization has converged, the band structure was calculated using a finer mesh of $\mathrm{k}$-points.

We find that the equilibrium position of the nitrogen ion is almost unchanged with respect to the corresponding $\mathrm{C}$ atom in the undoped nanotube, being moved by at most $0.01 \AA$, similarly to the case of azafullerene, $\mathrm{C}_{59} \mathrm{~N}$ 23, 24]. A slice of the calculated spin-density for both zigzag and armchair cases is shown in Fig. 1 The spin density is maximal on the $\mathrm{N}$ ion and the neighbouring $\mathrm{C}$ ions and shows oscillatory behaviour as it reaches a local maximum on every other ion in the network, again similarly to that observed in $\mathrm{C}_{59} \mathrm{~N}[23,24]$. The shape of the spin density isosurfaces is reminiscent of the $\pi$ orbitals in graphite with their axes of symmetry perpendicular to the sheet.

The localization of the extra electron associated with the isolated impurity shows a striking difference between

a)
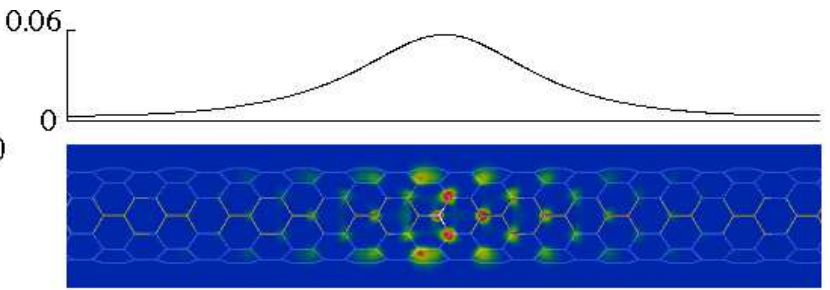

0.035$$
0
$$

b)

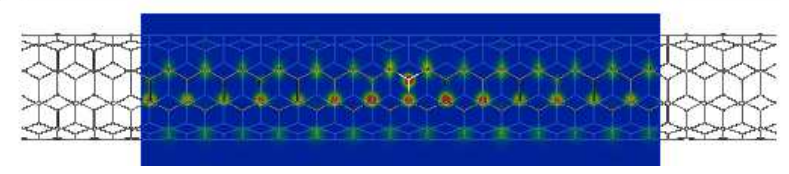

FIG. 1: Slice of the spin density of the N-doped carbon nanotube in a plane parallel to the tube axis, the plane being tangential to the tube at the position of the nitrogen ion, for a) $(8,0)$ zigzag tube, comprising 12 unit cells (50.6 $\AA$ ), and b) $(5,5)$ armchair tube, comprising 14 unit cells $(34.3 \AA$ ). Above each slice, we plot the envelope of the spin density (integrated in the plane perpendicular to the tube) in the units of electron/A. The nitrogen ion is marked white. The colour range maps the value of the spin density, with blue corresponding to the smallest and red to the highest values. Note that the scale of the spin density is quite different for the two panels. the metallic and semiconducting cases, despite the fact that the band gap of the latter is quite small $(0.6 \mathrm{eV} \mathrm{ac}-$ cording to the DFT calculations). In the armchair tube, the unpaired electron is almost completely delocalized along the tube, as can be seen from the plot of the envelope of the spin density in the lower panel of Fig. [1 However, for the zigzag tube, it is localized near the impurity site, but the localization region is fairly large, about 30 $\AA$. This region contains about $90 \%$ of the unpaired spin density. The decaying envelope of the spin density is exponential.

The oscillations in the spin density can be understood as follows. Regarding the nitrogen atom as perturbation, the response of the system is characterized by the static susceptibility $\chi(r)$. The characteristic period in $\chi(r)$ corresponds to a maximum in the Fourier transform, $\chi(q)$, which happens for $q$ values that connect points in the Brillouin zone with the minimum energy difference. For the $2 \mathrm{D}$ graphene lattice, the energy gap is zero at the high symmetry K points, so for this, and similar systems, $\chi(r)$ will oscillate with a period corresponding to the momentum transfer between different $\mathrm{K}$ points, which is what is observed. The well known oscillations in the susceptibility in the RKKY formalism 25] are essentially a special case of the above considerations.

The decay of the envelope of the spin density is also analogous to the RKKY case. For metals in 1D, the result is $\chi(r) \propto 1 / r$ 27, 28]. For semiconductors, an analogous coupling to excited states takes place, and assuming a parabolic conduction band, it was shown [26] that $\chi(r) \propto$ $\exp \left[-\left(\alpha E_{\text {gap }}^{1 / 2}\right) r\right]$, where $\alpha=\left(2 m_{\mathrm{eff}}\right)^{1 / 2} / \hbar$. For the zigzag nanotube, using the calculated value of $m_{\mathrm{eff}} / m_{e}=0.2$ this crude estimate gives a decay constant of about $6 \AA$, close to the observed $7 \AA$.

Note that in all the graphene-based systems $\left(\mathrm{C}_{59} \mathrm{~N}\right.$, nitrogen doped graphene and nanotubes), the spin density is positive almost everywhere. At first sight, this might seem to contradict the results from electron spin resonance hyperfine measurements, which indicate that the spin density oscillates symmetrically around zero. In fact, as was mentioned above, the spin density is very strongly dominated by the $p$ component, whereas the Fermi contact interaction in the hyperfine coupling is only sensitive to the $s$ component, so its observed symmetrical oscillation is consistent with the calculations.

A crucial difference between the armchair and the zigzag tubes is that the introduction of the nitrogen atom breaks the left-right mirror symmetry in the latter case. As two impurity atoms approach each other in an armchair tube, their interaction will show oscillatory behaviour depending on whether the spin density maxima induced by the respective nitrogens coincide or not. But in the zigzag tube the local maxima of the spin density occur on different sublattices on the two sides of the nitrogen atom. As two impurities approach, their respective spin density maxima never coincide, irrespective of 

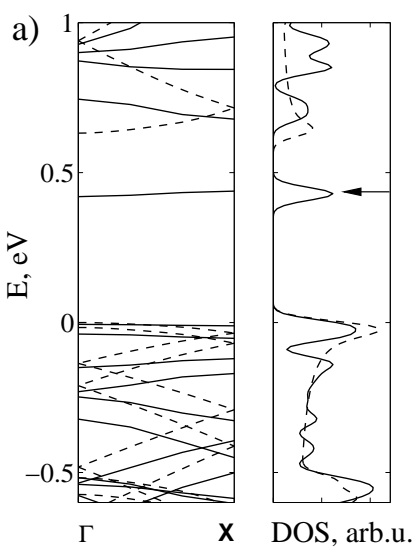

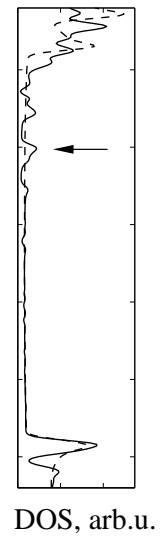

FIG. 2: Band structures and corresponding densities of states for the largest N-doped carbon nanotubes studied (solid line) versus the folded band structure of pure nanotube (dashed line) in a) $(8,0)$ zigzag nanotube, bands aligned in such a way that the valence band edge coincides with that of pure nanotube, and b) $(5,5)$ armchair tube, bands aligned in such a way that the van Hove singularities below the Fermi level coincide. The arrows mark the new band associated with the impurity. For reasons of clarity we only show the upspin channel, which contains the unpaired electron. Note that below the Fermi level, the splitting of the spin channels is less than $0.03 \mathrm{eV}$.

whether the nitrogens occupy the same sublattice or not.

Figure 2 shows the band structure of the nitrogendoped nanotubes along with the corresponding densities of states. The band structure of undoped tube (folded in such a way, that it fits into the shrinked Brillouin zone corresponding to the larger supercell) is shown for comparison. For the zigzag case, the extra band of the impurity falls somewhere near the bottom of the conduction band, and a complicated hybridization takes place with the existing unoccupied bands. The end result is that a new flat level appears about $0.2 \mathrm{eV}$ below the bottom of the conduction band. In the armchair tube, the impurity introduces a peak at about $0.6 \mathrm{eV}$ below the first van Hove singularity above the Fermi level, as well as a number of additional peaks, which are formed because of the hybridization and level repulsion taking place between the energy levels of impurity and pristine nanotube.

The localized electronic state of the semiconducting tube offers the intriguing possibility of being chemically active and forming covalent bonds with ligands or other tubes. Figure 3 shows two zigzag nanotubes each doped with nitrogen (one per four unit cells) in such a way that two carbon atoms with originally significant spin density on them face each other. As the charge density slice shows, a covalent bond is formed between these atoms, and thus between the tubes. The two carbon atoms pop out of the tangent plane of the tubes, and take up an $s p^{3}$ configuration, the bond length between them being

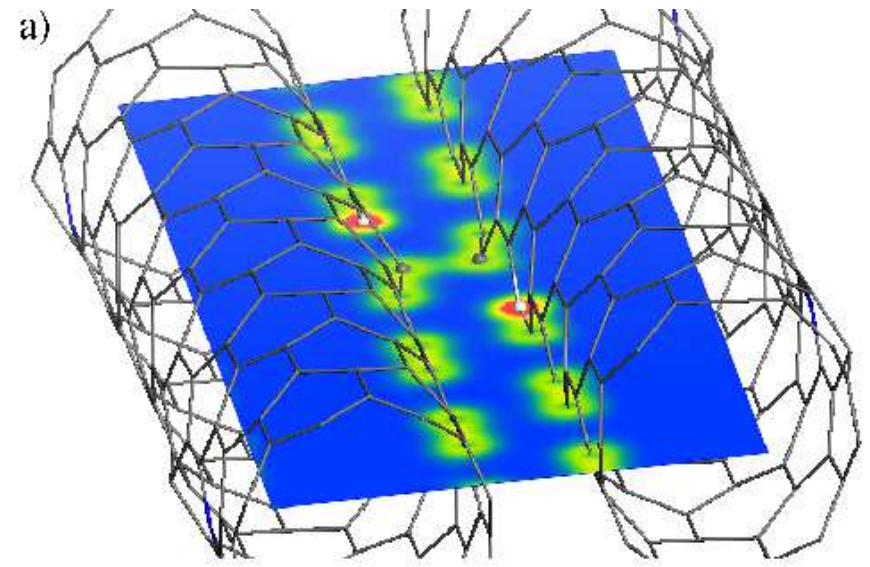

b)

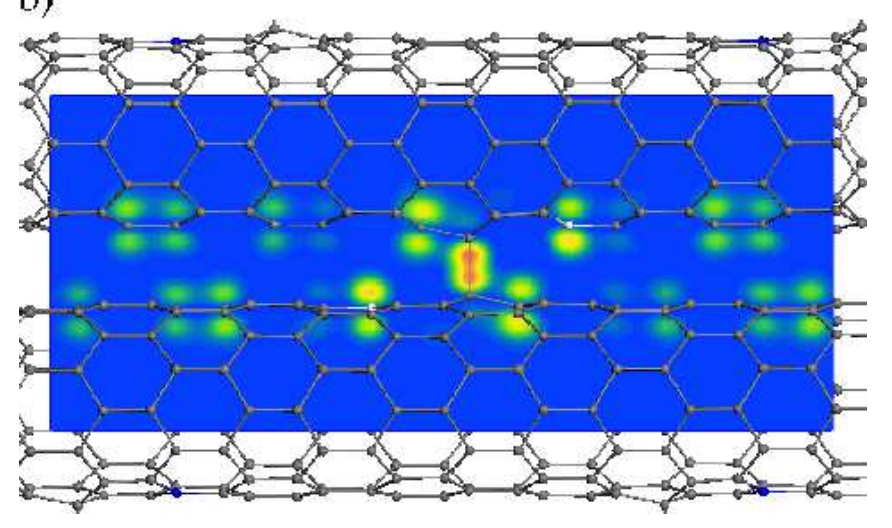

FIG. 3: Two zigzag nanotubes each doped with nitrogen (white balls), facing each other. The chemical bond is formed between the two carbon atoms (grey balls), which have the maximum of the spin density in the isolated nanotube. The slice taken through the $\mathrm{C}-\mathrm{C}$ bond shows the distribution of the a) total charge density and b) density of the HOMO orbital, using the colour map, with blue corresponding to the lowest and red to the highest densities.

$1.65 \AA$ (note that a similar geometry was discussed in the context of carbon nitride materials 29$]$ ). In order to obtain the geometry shown, the two tubes were pressed against each other, which is realistic when modelling a nanotube lying on top of another on a surface 30 . The further corroboration of the formation of a true covalent bond between the carbon atoms comes from the analysis of the charge density originating from different orbitals. The largest contribution to the total charge density between the newly bonded carbon atoms comes from the HOMO orbital (Fig. 3 3 ), which is occupied by about 0.3 of an electron.

On the other hand, for the intertube bonds to be realized in a bundle of SWNTs, they have to be stable under zero external pressure. We found that this is possible if the number of intertube bonds is increased to one per two unit cells $(8.4 \AA)$, so that enough energy is gained in the new bonds to overcome the intertube repulsion. Here, the bonded configuration is a local minimum and 

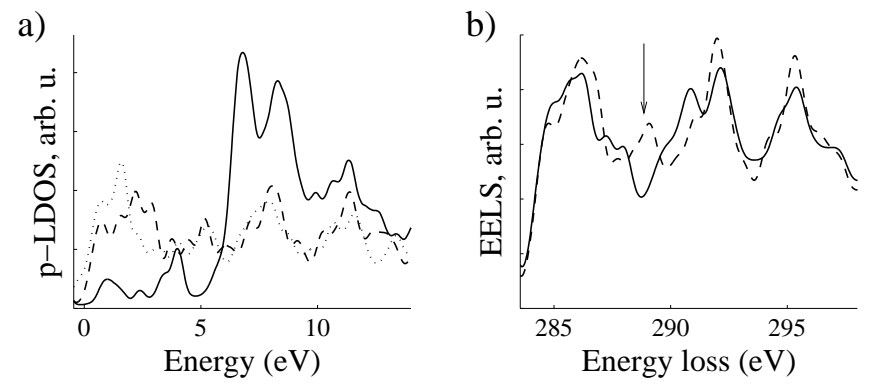

FIG. 4: a) $p$ projections of local densities of states at different atoms: $s p^{3}$ carbon atom in the $\mathrm{C}-\mathrm{C}$ bond (solid line), $s p^{2}$ carbon atom in the same tube, but far away from the bond (dotted line), $s p^{2}$ carbon atom in the unbonded isolated nanotube (dashed line); b) calculated EELS spectrum near the carbon $\mathrm{K}$ edge of bonded (solid line) and unbonded (dotted line) tubes, which is proportional to the $p$ projected total density of states, shifted appropriately.

the tube-tube distance is reduced to $2.5 \AA$, from $3.4 \AA$ An the case of the unbonded bundle. This finding opens the possibility of producing highly packed crosslinked bundles of doped nanotubes; we obtained a crude estimate of $80 \mathrm{GPa}$ for the bundle shear modulus (as a result of just one interlink for every 2 unit cells), which is substantially larger than 1-6 GPa, reported for ordinary bundles 31.

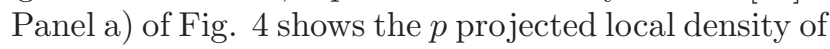
states (DOS) of various carbon atoms in different sections of the nanotube with one intertube bond per 2 unit cells, as compared to that of the unbonded system. The dashed curve shows the fine structure of the $\pi^{*}$ peak, which is almost completely absent for the $s p^{3}$ carbon atom (solid curve) that takes part in the intertube bond. As a first approximation, the $p$ projected total DOS in the conduction band, when suitably shifted, is proportional to the electron energy loss spectrum (EELS) [32, 33], and shown on panel b). In the bonded system the $\pi^{*}$ peak is somewhat diminished, but more importantly, the peak marked with an arrow is absent. Since the proportion of $s p^{3}$ bonded carbon atoms is expected to be quite small, a local probe such as EELS would be most suitable for the detection of the intertube bond.

We performed ab initio calculations of zigzag and armchair carbon nanotubes substitutionally doped with nitrogen. In the case of the armchair nanotube, the impurity state is totally delocalized and the corresponding energy level falls inside the unoccupied bands of the pristine nanotube. In the case of the semiconducting zigzag nanotube, however, the isolated nitrogen impurity forms a flat energy level lying inside the band gap, $0.2 \mathrm{eV}$ below the bottom of conduction band. It hybridizes with the $\pi$ orbitals to create a spatially localized state decaying exponentially with an overall extent of about $30 \AA$. This singly occupied state is chemically active, and we demonstrate the possibility of the formation of a covalent bond between two nanotubes with impurities that face one another. This opens the intriguing general possibility for chemical bonding between suitably doped carbon nanotubes or indeed with other ligands. Such tube-tube junctions could be interesting from a device perspective as the intertube bond will probably change the tunneling properties between the tubes considerably. If the density of intertube bonds is high enough, a bundle of SWNTs can become highly interlinked and packed, substantially enhancing its mechanical properties. The ligand-binding properties of the localized impurity site, of interest in chemical sensing applications, are currently under investigation.

The authors would like to thank Peter Littlewood, Ben Simons, David Khmelnitskii, Malcolm Heggie, Bill Allison and Howard Hughes for valuable discussions. Computational work was carried out at the HPCF, University of Cambridge and supported by grant EC HPRN-CT2000-00154.

[1] A. Oberlin, M. Endo, and T. Koyama, Carbon 14, 133 (1976); J. Cryst. Growth 32, 335 (1976)

[2] S. Iijima, Nature (London) 354, 56 (1991).

[3] R. Saito, G. Dresselhaus, and M.S. Dresselhaus, Physical Properties of Carbon Nanotubes (Imperial College Press, London, 1998).

[4] M. Yudasaka et al., Carbon 35, 195 (1997).

[5] R. Sen et al., Chem. Phys. Lett. 287, 671 (1998).

[6] M. Terrones et al., Appl. Phys. Lett. 75, 3932 (1999).

[7] N. Grobert et al., Appl. Phys. A 70, 175 (2000).

[8] W. Q. Han, Appl. Phys. Lett. 77, 1807 (2000).

[9] R. Kurt, J. M. Bonard, and A. Karimi, Thin Solid Films 398-399, 193 (2001); R. Kurt et al., Carbon 39, 2163 (2001).

[10] R. Czerw et al., Nano Letters 1, 457 (2001).

[11] M. Terrones et al., Appl. Phys. A 74, 355 (2002).

[12] S. Trasobares et al., J. Chem. Phys. 116, 8966 (2002).

[13] X. Wang et al., J. Phys. Chem. B 106, 2186 (2002).

[14] C. J. Lee et al., Chem. Phys. Lett. 359, 115 (2002).

[15] O. Stephan et al., Science 266, 1683 (1994).

[16] D. Golberg et al., Carbon 38, 2017 (2000); D. Golberg et al., Diam. and Related Mater. 10, 63 (2001).

[17] C. C. Kaun et al., Phys. Rev. B 65, 205416 (2002).

[18] J.-Y. Yi and J. Bernholc, Phys. Rev. B 47, 1708 (1993).

[19] P. E. Lammert, V. H. Crespi, and A. Rubio, Phys. Rev. Lett. 87, 136402 (2001).

[20] M.C. Payne et al., Rev. Mod. Phys. 64, 1045 (1992).

[21] V. Milman et al., Int. J. Quant. Chem. 77, 895 (2000).

[22] J. P. Perdew, K. Burke, and M. Ernzerhof, Phys. Rev. Lett. 77, 3865 (1996);

[23] W. Andreoni, F. Gygi, and M. Parrinello, Chem. Phys. Lett. 190, 159 (1992).

[24] G. Csanyi and T. A. Arias, Chem. Phys. Lett. 360, 552 (2002). 78, 1396(E) (1997).

[25] M. A. Ruderman and C. Kittel, Phys. Rev. 96, 99 (1954); T. Kasuya, Prog. Theor. Phys. (Kyoto) 16, 45 (1956); K. Yosida, Phys. Rev. 106, 893 (1957).

[26] N. Bloembergen and T. Rowland, Phys. Rev. 97, 1679 
(1955)

[27] D. N. Aristov, Phys. Rev. B 55, 8064 (1997).

[28] V. I. Litvinov and V. K. Dugaev, Phys. Rev. B. 58, 3584 (1998).

[29] É. Sandré, C. J. Pickard, and C. Colliex, Chem. Phys. Lett. 325, 53 (2000).

[30] M.S. Fuhrer et al., Science 288, 494 (2000).
[31] J.-P. Salvetat, et al., PRL 82, 944 (1999).

[32] P. Rez, J. R. Alvarez, and C. Pickard, Ultramicroscopy 78, 175 (1999).

[33] R. F. Egerton, Electron Energy-Loss Spectroscopy in the Electron Microscope (Plenum Press, New York-London, 1996). 